\title{
Functionalized Single Graphene Sheets Derived from Splitting Graphite Oxide
}

\author{
Hannes C. Schniepp,${ }^{\dagger}$ Je-Luen Li, ${ }^{\ddagger}$ Michael J. McAllister, ${ }^{\dagger}$ Hiroaki Sai, ${ }^{\dagger}$ \\ Margarita Herrera-Alonso, ${ }^{\dagger}$ Douglas H. Adamson, ${ }^{\S}$ Robert K. Prud’homme, ${ }^{\dagger}$ Roberto Car, ${ }^{\ddagger}$ \\ Dudley A. Saville, ${ }^{\dagger}$ and Ilhan A. Aksay*, ${ }^{*}$ \\ Department of Chemical Engineering, Department of Chemistry, and Princeton Institute for the Science and \\ Technology of Materials (PRISM), Princeton University, Princeton, New Jersey 08544
}

Received: February 13, 2006; In Final Form: March 16, 2006

\begin{abstract}
A process is described to produce single sheets of functionalized graphene through thermal exfoliation of graphite oxide. The process yields a wrinkled sheet structure resulting from reaction sites involved in oxidation and reduction processes. The topological features of single sheets, as measured by atomic force microscopy, closely match predictions of first-principles atomistic modeling. Although graphite oxide is an insulator, functionalized graphene produced by this method is electrically conducting.
\end{abstract}

\section{Introduction}

Recent studies ${ }^{1-6}$ of the electrical properties of graphene sheets produced by peeling off a few sheets with "Scotch" tape demonstrate the importance of graphene as a $2 \mathrm{D}$ crystalline material for high electron mobility applications. ${ }^{7}$ Moreover, when functionalized, single-sheet graphene is expected to serve as a nanofiller in nanocomposite applications, as functional sites will favor interactions with the matrix. ${ }^{8}$ The first attempt to produce individual graphene sheets by exfoliation dates to the work of Brodie in 1859. ${ }^{9}$ Since then, and despite many attempts, ${ }^{10-17}$ large-scale production of single graphene sheets has not been achieved. In most studies, the starting material is graphite oxide (GO) produced through an acid treatment of graphite. GO is then exposed to either a thermal or mechanical (i.e., ultrasonication) treatment to expand or to exfoliate it. Although nanoplates of a few sheets have been generated by the solution approach, ${ }^{17,18}$ the failure to produce single sheets by thermal expansion appears to be either due to insufficient oxidation of graphite during the acid treatment or inadequate pressure buildup during the thermal heat treatment stages. In this Letter, we show that, through an optimal combination of GO preparation and thermal treatment, bulk quantities of functionalized single graphene sheets can be produced. The mechanism of exfoliation is mainly the expansion of $\mathrm{CO}_{2}$ evolved into the interstices between the graphene sheets during rapid heating. While the decomposition of functional groups of $\mathrm{GO}$ yielding $\mathrm{CO}_{2}$ is exothermic, the vaporization of water is endothermic and delays the heating process. For the success of the process, it is essential to completely eliminate the intergraphene spacing associated with the native graphite during the oxidation stage and also to minimize the detrimental role of water vaporization.

\footnotetext{
*Corresponding author. E-mail: iaksay@ princeton.edu.

${ }^{\dagger}$ Department of Chemical Engineering, Princeton University.

Department of Chemistry, Princeton University.

$\S$ Princeton Institute for the Science and Technology of Materials (PRISM), Princeton University.
}

An NMR study ${ }^{19}$ has shown that GO as used in the thermal exfoliation process contains aromatic regions randomly interspersed with oxidized aliphatic six-membered rings. The oxidized rings contain $\mathrm{C}-\mathrm{O}-\mathrm{C}$ (epoxide) and $\mathrm{C}-\mathrm{OH}$ groups, while the sheets terminate with $\mathrm{C}-\mathrm{OH}$ and $-\mathrm{COOH}$ groups. Some of these functional groups are retained in the thermally exfoliated nanoplates of graphene stacks. Thus, when used as a nanofiller in a polymer (poly(methyl methacrylate)) matrix, these functionalized graphene stacks offer comparable or better thermal, mechanical, and electrical property enhancements than SWCNTs. ${ }^{8,20}$

\section{Experimental Methods}

We begin by treating natural flake graphite, nominally $\sim 45$ $\mu \mathrm{m}$ across (Asbury Carbons, Asbury, NJ), in an oxidizing solution of sulfuric acid, nitric acid, and potassium chlorate (for $>96 \mathrm{~h}$ ), similar to a process first described in 1898 (SI1, Supporting Information). ${ }^{10}$ The fundamental prerequisites that enable our preparation of large volumes of dry single graphene sheets are complete oxidation of graphite and extremely rapid heating of GO. Complete oxidation is monitored by the total disappearance of the 0.34 -nm intergraphene spacing and the appearance of a new one with a $0.65-0.75 \mathrm{~nm}$ range depending on the water content of GO, as determined by X-ray and electron diffraction (SI1, Supporting Information). In terms of structure, the disappearance of the $0.34 \mathrm{~nm}$ has been reported previously, ${ }^{18}$ but GO with such characteristics has never been used in thermal expansion studies, which we find to be an essential step for a successful splitting of GO into single graphene sheets. To quantify the amount of residual water and the evolved gases, we use simultaneous thermal gravimetric analysis (TGA), differential scanning calorimetry (DSC), and Fourier transform infrared (FTIR) spectroscopy. For thermal exfoliation, the dried graphite oxide is charged into a quartz tube and purged with argon. Rapid heating $\left(>2000{ }^{\circ} \mathrm{C} / \mathrm{min}\right)$ to $1050{ }^{\circ} \mathrm{C}$ splits the graphite oxide into individual sheets through evolution of $\mathrm{CO}_{2}$ (SI1, Supporting Information). Elimination of all the diffraction 
peaks (associated with the initial GO stacking) after rapid heating signifies a successful exfoliation. To quantify the extent of exfoliation, we measure the surface areas of the products (i) by the Brunauer, Emmett, and Teller $(B E T)^{21}$ method with nitrogen gas adsorption in the dry state and (ii) in an ethanol suspension with methylene blue dye as a probe. Methylene blue has been used as an indicator of graphitic material surface areas in previous studies, with each milligram of adsorbed methylene blue representing $2.45 \mathrm{~m}^{2}$ of surface area. ${ }^{22}$ However, the ambiguities in surface areas determined by BET and methylene blue adsorption (SI2, Supporting Information) led us to rely on atomic force microscopy (AFM) measurements to confirm the presence of single sheets.

For AFM characterization, the flakes were dispersed in 1-methyl-2-pyrrolidinone (NMP) and spin-coated onto highly oriented pyrolytic graphite (HOPG) (SI3, Supporting Information). The samples were examined by using a MultiMode/ NanoScope IIIa AFM (Veeco Metrology LLC, Santa Barbara, CA). Tapping-mode imaging was applied to provide the largest amount of structural detail of the graphene sheets as well as to prevent translocation of the sheets on the surface by the tip (Veeco MP-11100 silicon cantilevers, force constant $k=40$ $\mathrm{N} / \mathrm{m}$, radius of curvature $r=10 \mathrm{~nm}$, and resonance frequency $f=300 \mathrm{kHz}$ ). For precise measurement of the flake thickness, contact-mode imaging was performed (Veeco NP-S type, oxidesharpened and gold-coated silicon nitride cantilevers, $k=0.32$ $\mathrm{N} / \mathrm{m}, r=20 \mathrm{~nm}$ ). For height analysis, topography sections across a sheet, starting and ending on the HOPG substrate, were taken, following the fast scanning direction in order to minimize the effect of drifts.

The molecular modeling was done using Car-Parrinello simulations ${ }^{23}$ as implemented in Quantum-ESPRESSO distribution. ${ }^{24}$ To investigate the structural properties of functional groups, vacancies, and topological defects on a carbon grid, we employed a small planar-fused benzene ring $\left(\mathrm{C}_{54} \mathrm{H}_{12}\right)$ and an extended long strip of graphene, where the sides are terminated by hydrogen atoms. The structures were relaxed until the force on each atom was less than $0.2 \mathrm{eV} / \mathrm{nm}$. In particular, we retained a large vacuum region of at least $1.3 \mathrm{~nm}$ around $\mathrm{C}_{54} \mathrm{H}_{12}$ and the two free sides of the graphene strip so that they can bend during geometry optimization.

\section{Results and Discussion}

The indicators of a successful exfoliation process are: (i) a 500-1000-fold volume expansion after the rapid heat treatment of GO, (ii) the disappearance of all the diffraction peaks after the rapid heat treatment, and (iii) surface areas in the range of $700-1500 \mathrm{~m}^{2} / \mathrm{g}$ as determined by the BET adsorption method (SI1 and SI2, Supporting Information). When GO was not adequately dried, lower surface areas resulted (SI2, Supporting Information). Significantly higher surface areas $(\sim 2 \times)$ were measured using methylene blue adsorption ${ }^{22}$ on graphene suspended in ethanol.

We associate the exfoliation process with the thermal expansion of the evolved gases trapped between the graphene sheets. We also associate the detrimental effect of water to the fact that, while the decomposition of functional groups is exothermic, vaporization of water is endothermic and thus slows down the heating process (SI1, Supporting Information). We estimate the van der Waals attraction between the graphite layers as $26 \mathrm{MPa}$ by using a Lennard-Jones potential and pairwise summation. The maximum pressures generated by both water and carbon dioxide estimated by using the spacing of the layers from diffraction measurements are determined to be in excess of 60 and $100 \mathrm{MPa}$ for water and $\mathrm{CO}_{2}$, respectively (SI2, Supporting Information). Both pressures exceed the van der Waals attraction between the graphite layers. However, the $\mathrm{CO}_{2}$ evolution, corresponding to a weight loss of $30 \%$, is the predominant mechanism driving exfoliation (SI1, Supporting Information).

As shown in the AFM images of Figure 1a, the exfoliated sheets are well dispersed at an average density of about 50 flakes per $10 \mu \mathrm{m} \times 10 \mu \mathrm{m}$ area and exhibit a lateral extent of a few hundred nanometers. A higher magnification image of a representative flake (Figure $1 \mathrm{~b}$ ) reveals that the sheets display height variations at two length scales: (i) the flat areas of the sheet have an average height of about $2 \mathrm{~nm}$ with respect to the HOPG surface and are covered with $\sim 0.2-0.4 \mathrm{~nm}$ "bumps" (Figure $1 \mathrm{~b}$ and d), and (ii) several large, meandering wrinkles with peak heights up to $10 \mathrm{~nm}$ (Figure 1b). Extensive analysis of the AFM data shows that all of the flakes examined are single sheets (vide infra). Below, we relate the bumpy texture of the flat regions to the presence of isolated epoxy and hydroxyl reaction sites. We also show that the wrinkles correspond to defects in the carbon lattice as remnants of the epoxy reaction strings.

Results of our first-principles atomistic model of two oxygencontaining functional epoxy and hydroxyl groups shown in Figure $2 \mathrm{a}$ and $\mathrm{b}$ indicate that these groups introduce an additional $\sim 0.44 \mathrm{~nm}$ of height to the carbon grid. The carbon atom functionalized by an epoxy or a hydroxyl group is transformed from a planar $\mathrm{sp}^{2}$-hybridized to a distorted $\mathrm{sp}^{3}$-hybridized geometry. The protuberant carbon atom is 0.03 and $0.07 \mathrm{~nm}$ above the carbon grid for epoxy and hydroxyl functional groups, respectively. The functional groups themselves are $\sim 0.2 \mathrm{~nm}$ above the underlying carbon grid, as shown in Figure $2 a$ and $b$. As shown in Figure 2c, when functional groups randomly bind to both sides of a small graphene platelet, there is no largescale height variation. The $0.44-\mathrm{nm}$ increase in height correlates well with the increase in the separation distance of the graphene layers (from 0.34 to a $0.65-0.75 \mathrm{~nm}$ range, as determined by $\mathrm{X}$-ray and electron diffraction) when graphite is transformed into graphite oxide. A C/O ratio of 10:1, after the thermal treatment, indicates that some functional groups are still on the graphene sheets (SI4, Supporting Information). Thus, the smaller bumps of $\sim 0.2-0.4 \mathrm{~nm}$ discussed above (Figure $1 \mathrm{~b}$ and $\mathrm{d}$ ) agree well with the prediction of our model.

To interpret the larger wrinkles peaking at heights of $\sim 10$ $\mathrm{nm}$, we consider the specifics of both the oxidation and the reduction during the exfoliation process. Unlike functional groups such as hydroxyls or carboxyls, epoxy groups generate strain on the $\mathrm{C}-\mathrm{C}$ bond of the three-membered epoxide ring. According to a recently proposed mechanism, when several epoxy groups line up, the sheet can form a kink that partially releases this strain energy and makes this configuration energetically favored. ${ }^{25}$ Therefore, during the oxidation of graphite, epoxy groups tend to form chains across the graphite surface through this cooperative reaction (Figure $3 a$ and $a^{\prime}$ ). Because of the elimination of oxygen from functional groups and any residual water during the rapid heating, elemental analysis shows an increase in the C/O ratio from 2:1 in graphite oxide to 10:1 in the reduced state (SI4, Supporting Information). While the expansion of intercalated water to vapor phase along with $\mathrm{CO}_{2}$ evolution was once thought to be responsible for the exfoliation process, the presence of water is in fact detrimental due to the cooling effect of water evaporation (SI2, Supporting Information). Instead, the pressure needed to counteract the van der Waals binding energy holding the graphene sheets together and split graphite oxide into single graphene sheets (SI2, Supporting 

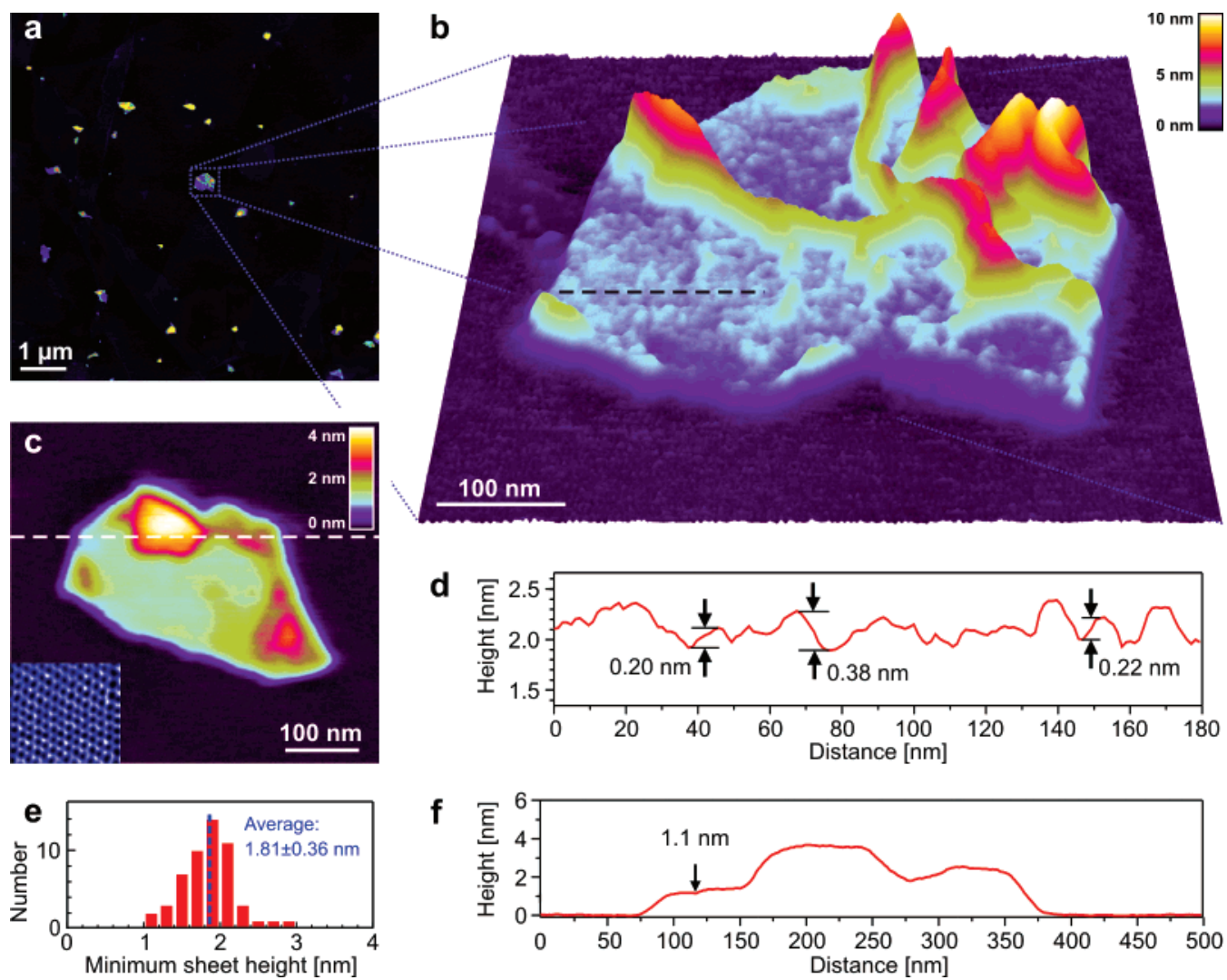

Figure 1. (a) $8 \mu \mathrm{m} \times 8 \mu \mathrm{m}$ tapping-mode AFM topography image showing individual thermally exfoliated graphite oxide flakes. (b) Pseudo-3D representation of a $600 \mathrm{~nm} \times 600 \mathrm{~nm}$ AFM scan of an individual graphene sheet showing the wrinkled and rough structure of the surface. (c) Contact-mode AFM scan of a different flake on the same sample, providing the most accurate thickness measurement of the sheet as well as an atomic-scale image of the HOPG lattice (inset). (d) Cross-section taken at a rough, unwrinkled area on top of the sheet shown in (b) (position indicated by black dashed line in (b)). (e) Histogram showing the narrow distribution of minimal sheet heights measured on 53 different sheets. (f) Cross-section through the sheet shown in (c) exhibiting a height minimum of $1.1 \mathrm{~nm}$.
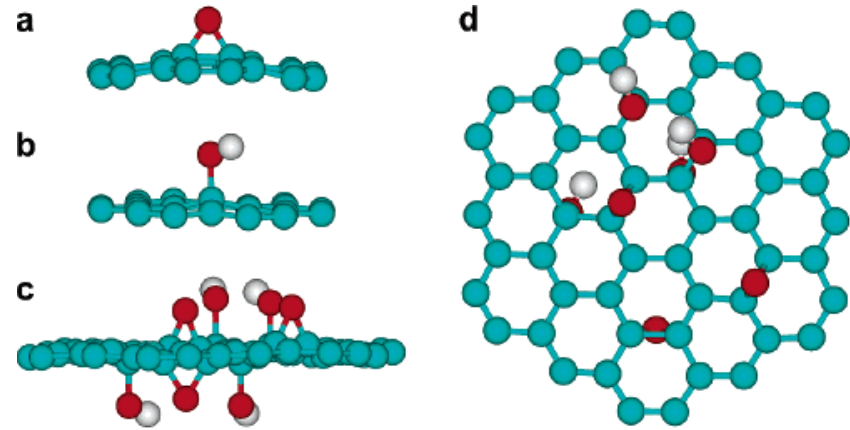

Figure 2. (a) An epoxy group. The oxygen atom (red colored) is 0.19 $\mathrm{nm}$ above the carbon grid. (b) A hydroxyl group. The top hydrogen atom is $0.22 \mathrm{~nm}$ above the carbon grid. (c) Edge-on view of a graphene sheet with functional sites such as epoxy and hydroxyl groups on both sides. The oxygen atom (red colored) is $0.19 \mathrm{~nm}$ above the carbon grid. The top hydrogen atom (grey colored) of the hydroxyl group is $0.22 \mathrm{~nm}$ above the carbon grid. (d) The top view of (c). Together with the backbone carbon grid, the functional groups constitute a single layer of graphite oxide with a thickness of $\sim 0.78 \mathrm{~nm}$.

Information) is furnished by $\mathrm{CO}_{2}$ evolved by (exothermic) reactions involving epoxy and hydroxyl sites. The $\mathrm{CO}_{2}$ release during this expansion corresponds to $\sim 30 \mathrm{wt} \%$ loss. The residual material still contains oxygen, as confirmed by X-ray photoelectron spectroscopy (XPS) and elemental analysis (SI4, Supporting Information).

The release of carbon dioxide inevitably leaves behind vacancies and topological defects on the graphene sheets. In our molecular simulation, two carbon atoms and four epoxy groups are removed from the small graphene platelet, $\mathrm{C}_{54} \mathrm{H}_{12}$.
Two single-carbon vacancies then coalesce into a 5-8-5 double vacancy $^{26}$ (SI5, Supporting Information) and result in a buckled structure, as shown in Figure 3b. Parts c and d of Figure 3 juxtapose the same graphene sheet with a line defect (a buckling fault line). We propose that the experimentally observed wrinkles at a larger length scale (Figure 1b and 3d) display remnants of these linear carbon deficiency patterns. According to the molecular modeling, the sides (Figure 3c) are bent down by $21.5^{\circ}$ (Figure 3d). Figure 3c represents one such pattern; other compositions, with or without single vacancy and/or epoxy groups, were also studied and showed similar bending angles. To compare the model with our experimental data, Gaussians are fitted to sections across wrinkles of Figure $1 \mathrm{~b}$ and other sheets at several positions, and the corresponding maximal slope angles were determined (typical experimental values and fits are shown in Figure 3d). We find similar slope angles for wrinkle heights between 2.4 and $9.5 \mathrm{~nm}$, which suggests that the slope angle is dictated by topological defects lined up along the wrinkle; the average value of $20 \pm 5^{\circ}$ is in good agreement with the model. When isolated reaction sites decompose, bending is also expected due to the formation of defects, but only as enhancements to the bumpy texture by the functional sites and thus increasing the apparent height of the graphene sheets further.

To demonstrate that the majority of our material consists of single layers of functionalized graphene, we analyzed AFM topography cross-sections. Because of the wrinkling and buckling of the sheets, only a small fraction of each sheet is in contact with the substrate. We therefore take the minimum height of every sheet with respect to the substrate as an estimate 

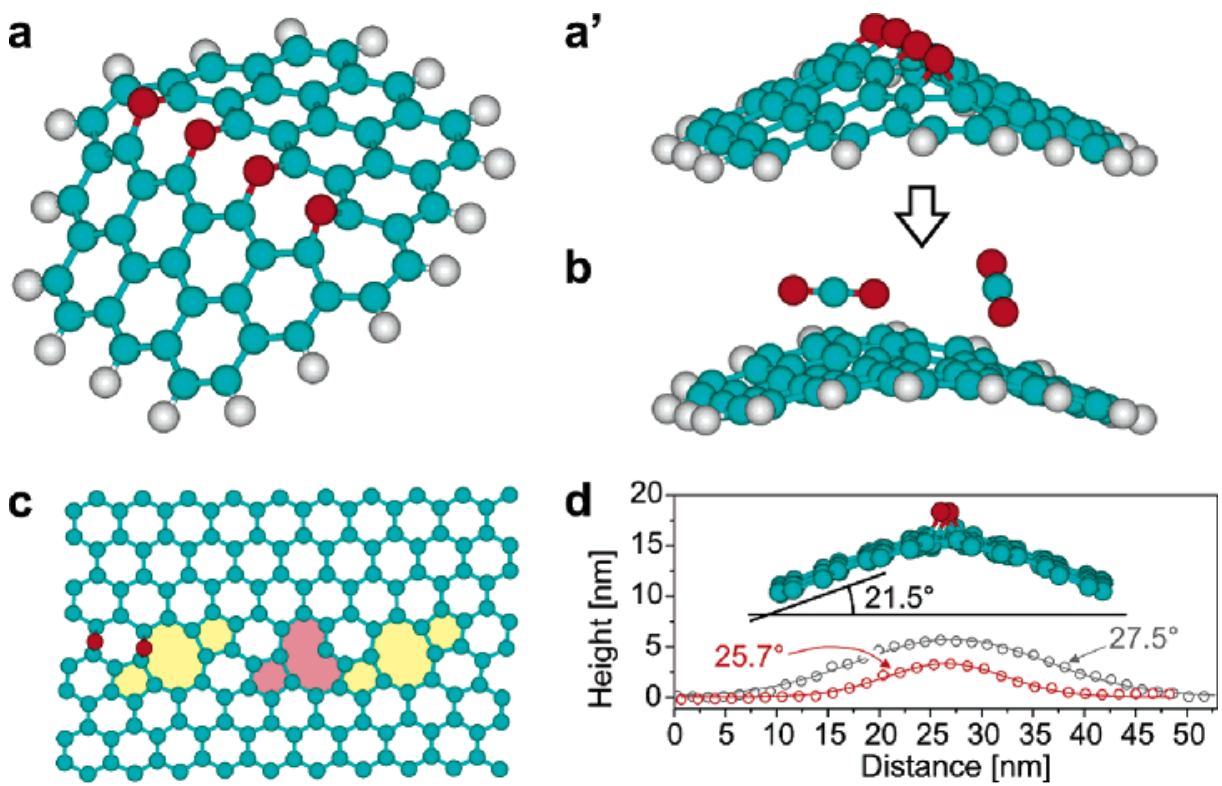

Figure 3. Atomistic model of the graphite oxide to graphene transition. (a, a') During oxidization of graphite, linear clusters of epoxy groups are formed through cooperative binding that lead to a bent sheet. (b) Carbon dioxide is released during thermal treatment and leaves vacancies and topological defects on the carbon grid that remains bent after reduction. Here we show a 5-8-5 defect, which is created from coalescence of two single-carbon vacancies left behind after reduction process (see SI5, Supporting Information). (c) A long strip of graphene sheet with a line defect. The two 5-8-5 defects are labeled in yellow and a single-carbon vacancy with a nearby pentagon ring is labeled in pink. Also shown on the left are two epoxy groups aligned in parallel. (d) Comparison between AFM profiles across wrinkled structures and the curvature of the buckled graphene strip of (c). The open circles represent cross-sections through two wrinkles of different height in Figure 1b. The lines are corresponding Gaussian fits. Note that we offset the atomistic model to assist comparison.

of its thickness. Parts $\mathrm{c}$ and $\mathrm{f}$ of Figure 1 juxtapose a contactmode image and a corresponding cross-section. The height of the sheet in Figure 1c with respect to the HOPG substrate is as low as $1.1 \mathrm{~nm}$ in some areas, so this flake can only consist of a single sheet of functionalized graphene based on the interplanar GO spacing $d_{\mathrm{GO}}=0.65-0.75 \mathrm{~nm}$. Similar height analysis of minimum sheet thickness performed on 53 sheets (Figure 1e) yields an average value of $1.81 \pm 0.36 \mathrm{~nm}$. On the basis of the value of $d_{\mathrm{GO}}$, the majority of the sheets must therefore consist of one to three layers of functionalized graphene. If the measured ensemble contained significant numbers of mixed species of single, double, and triple layer sheets, the width of the histogram in Figure 1e would have to be much larger than the observed $0.36 \mathrm{~nm}$. Therefore, the majority of the sheets in this ensemble consist of the same number of layers. There is, however, no known process that would mainly form either double or triple layer sheets without yielding a significant amount of single sheets. We thus contend the most plausible scenario is that complete exfoliation is achieved and all the investigated sheets consist of single-layer graphene. The fact that the averaged height minima of the sheets reflected by the histogram are higher than $d_{\mathrm{GO}}$ is due to following reasons: (i) roughness on scales smaller than the AFM probe $(\sim 20 \mathrm{~nm})$ is not detected by this measurement and will lead to an overestimate of the sheet thickness, as the tip cannot reach the lowest points in this case, and (ii) the value of $d_{\mathrm{GO}}$ is determined from layered bulk material. For a single functionalized graphene sheet on top of an HOPG substrate, however, the van der Waals force is reduced. ${ }^{27}$ For instance, an increased spacing has been observed for single graphene sheets on a $\mathrm{SiO}_{2}$ substrate. ${ }^{1,4}$

Despite the defective structure of the graphene sheets and some residual functional sites after the reduction step, dc conductivities of compacts at a bulk density of $\sim 0.3 \mathrm{~g} / \mathrm{cm}^{3}$ range from $1 \times 10^{3}$ to $2.3 \times 10^{3} \mathrm{~S} / \mathrm{m}$. This value compares favorably with conductivities of compressed graphite monoliths measured by Celzard et al. ${ }^{28}$ at similar bulk densities (SI6, Supporting Information).

\section{Conclusions}

As a filler in polymer nanocomposites, stacks of these graphene sheets have already yielded outstanding thermal, mechanical, and electrical properties, incorporating in one material the best features of both clay- and carbon nanotubebased nanocomposites. ${ }^{8}$ We anticipate that the functionalized single graphene sheets described here will provide even further benefits, not only for structural property enhancements at lower filler contents, but also for the development of new materials such as electrically conducting polymers and inks (SI6, Supporting Information) and ultracapacitors.

Acknowledgment. We thank Sibel Korkut for the data on the electrical conductivity (SI6) of the graphene material discussed in this Letter. Financial support from the NASA University Research, Engineering, and Technology Institute on BioInspired Materials (BIMat) under award no. NCC-1-02037 is greatly appreciated.

Supporting Information Available: Details on the following topics are available: production of functionalized graphene sheets, surface area measurements, sample preparation for AFM studies, compositional analysis, defect formation on graphite, and conductivity measurements. This material is available free of charge via the Internet at http://www.pubs.acs.org.

\section{References and Notes}

(1) Novoselov, K. S. et al. Science 2004, 306, 666 and the online material of this paper.

(2) Duplock, E. J.; Scheffler, M.; Lindan, P. J. D. Phys. Rev. Lett. 2004, 92, 225502 . 10451. 

201.

(5) Zhang, Y.; Small, J. P.; Amori, M. E. S.; Kim, P. Phys. Rev. Lett. 2005, 94, 176803 .

(6) Novoselov, K. S.; Geim, A. K.; Morozov, S. V.; Jiang, D.; Katsnelson, M. I.; Grigorieva, I. V.; Dubonos, S. V.; Firsov, A. A. Nature 2005, 438, 197.

(7) Wilson, M. Phys. Today 2006, 59, 21.

(8) Brinson, L. C.; Prud'homme, R. K.; Ramanathan, T.; Abdala, A. A. Stankovich, S; Dikin, D. A.; Herrera-Alonso, M. Piner, R. D. Adamson, D. H.; Liu, J.; Chen, X.; Ruoff, R. S.; Nguyen, S. T.; Aksay, I. A. in preparation.

9) Brodie, B. C. Philos. Trans. R. Soc. London 1859, 149, 249.

(10) Staudenmaier, L. Ber. Dtsch. Chem. Ges. 1898, 31, 1481.

(11) Hummers, W.; Offeman, R. E. J. Am. Chem. Soc. 1958, 80, 1339

(12) Boehm, H. P.; Clauss, A.; Fischer, G. O.; Hofmann, U. Z. Anorg. Allg. Chem. 1962, 316, 119.

(13) Boehm, H. P.; Scholtz, W. Z. Anorg. Allg. Chem. 1965, 335, 74

(14) Lueking, A. D.; Pan, L.; Narayanan, D. L.; Clifford, C. E. B. J. Phys. Chem. B 2005, 109, 12710

(15) Fukushima, H.; Drzal, L. T. Annu. Technol. Conf.-Soc. Plast. Eng. $\mathbf{2 0 0 3}, 61,2230$

(16) Matsuo, Y.; Higashika, S.; Kimura, K.; Mayamoto, Y.; Fukutsuka, T.; Sugie, Y. J. Mater. Chem. 2002, 12, 1592.

(17) Stankovich, S.; Piner, R. D.; Chen, X.; Wu, N.; Nguyen, S. T.; Ruoff, R. S. J. Mater. Chem. 2006, 16, 155.
(18) Du, X. S.; Xiao, M.; Meng, Y. Z.; Hay, A. S. Carbon 2005, 43,

(19) Lerf A.; He, H.; Forster, M.; Klinowski, J. J. Phys. Chem. B 1998, $102,4477$.

(20) Iijima, S.; Ichihashi, T. Nature 1991, 363, 603.

(21) Brunauer, S.; Emmett, P. H.; Teller, E. J. Am. Chem. Soc. 1938, 60, 309-19.

(22) Boehm, H. P.; Clauss, A.; Fisher, G. O.; Hofmann, U. Z. Anorg. Allg. Chem. 1962, 316, 119.

(23) Car, R.; Parrinello, M. Phys. Rev. Lett. 1985, 55, 2471.

(24) Quantum-ESPRESSO code, http://www.pswcf.org. We employed the PBE-GGA exchange-correlation functional (Perdew, J. P.; Burke, K.; Ernzerhof, M. Phys. Rev. Lett. 1996, 77, 3865) and ultrasoft pseudopotential (Vanderbilt, D. Phys. Rev. B 1990, 41, 7892). The electronic Kohn-Sham orbitals were expanded in a plane-wave basis set with an energy cutoff of 25 Ry.

(25) Li, J.-L.; Kudin, K. N.; McAllister, M. J.; Prud'homme, R. K.; Aksay, I. A.; Car, R. Phys. Rev. Lett. submitted.

(26) Lee, G.-D.; Wang, C. Z.; Yoon, E.; Hwang, N.-M.; Kim, D.-Y.; Ho, K. M. Phys. Rev. Lett. 2005, 95, 205501

(27) Parsegian, V. A. van der Waals Forces: A Handbook for Biologists, Chemists, Engineers, and Physicists; Cambridge University Press: New York, 2005; Equation L1.30.

(28) Celzard, A.; Marech, J. F.; Furdin, G.; Puricelli, S. J. Phys. D: Appl. Phys. 2000, 33, 3094. 